\title{
Strain Effects on Optical Properties of (In,Ga)As-Capped InAs Quantum Dots Grown by Molecular Beam Epitaxy on GaAs (113)A Substrate
}

\author{
Faouzi Saidi, Mouna Bennour, Lotfi Bouzaïene, Larbi Sfaxi, and Hassen Maaref \\ Laboratoire de Micro-Optoelectroniques et Nanostructures, Faculté des Sciences de Monastir, Université de Monastir, \\ Monastir 5000, Tunisia \\ Correspondence should be addressed to Faouzi Saidi, faouzi.saidi@fsm.rnu.tn
}

Received 14 July 2011; Revised 16 August 2011; Accepted 24 August 2011

Academic Editor: Kam-Sing Wong

Copyright () 2011 Faouzi Saidi et al. This is an open access article distributed under the Creative Commons Attribution License, which permits unrestricted use, distribution, and reproduction in any medium, provided the original work is properly cited.

We have investigated the optical properties of InAs/GaAs (113)A quantum dots grown by molecular beam epitaxy (MBE) capped by (In,Ga)As. Reflection high-energy electron diffraction (RHEED) is used to investigate the formation process of InAs quantum dots (QDs). A broadening of the PL emission due to size distribution of the dots, when InAs dots are capped by GaAs, was observed. A separation between large and small quantum dots, when they are encapsulated by InGaAs, was shown due to hydrostatic and biaxial strain action on large and small dots grown under specifically growth conditions. The PL polarization measurements have shown that the small dots require an elongated form, but the large dots present a quasi-isotropic behavior.

\section{Introduction}

Self-assembled quantum dots (QDs) have been studied intensively for more than a decade due to their unique physical properties arising from the three-dimensional quantum confinement of carriers and delta-like density of state [1-3]. QDs find many applications in optoelectronic devices [2,3], delivering a striking improvement of the performance over conventional technology. However, the stochastic nature of the QDs makes it difficult to obtain dots with uniformity in both their size and their spatial distribution, which constitute the most dramatic problem that prevents the production of optoelectronic devices with a high quality of dots since it is incompetent to provide the prospect of temperature-independent low-threshold lasers $[4,5]$. The structural and optical properties strongly depend on growth conditions, such as growth temperature, growth rate, and the capping layer material. One way to improve or adjust the QDs properties is the utilization of high-index substrates which exhibit some interesting phenomena, with respect to (001) orientation. To date, there are few reports about successful growth of self-organized QDs on high-index substrates, comparable to those with (001) orientation $[6,7]$.
Growth studies have also been realized with the intention of controlling size, shape, and density of the QDs [8]. The growth control and the valid results, obtained on these structures, which are elaborated on high-index substrates, have permitted to improve optical and electrical properties of many compounds $[9,10]$.

Prior to understanding how the capping layer influences the transition energies of $[11 k]$ grown InAs QDs, one has to know the effect on the transition energies of QD growth on $[11 k]$ substrates $(k=1,2,3)$. The origin of the variation of the transition energy with the substrate orientation can be traced back to the competition of several effects: (i) hydrostatic component of the strain tensor is responsible for a shift of the conduction band upwards and the valence bands downwards, (ii) biaxial component of the strain tensor influences the degree of the valence band mixing, and (iii) variation of the hole effective mass with the substrate orientation, which can significantly alter the effects of the size quantization in the QD. Theoretically, Mlinar et al. $[8,9]$ have shown that the QD size in the growth direction determine which of the three above-mentioned effects will be the dominant one, regardless of the dot shape. 
In this paper, we reported the experimental details of the photoluminescence spectroscopy on the InAs/GaAs (113) capped by GaAs and InGaAs epilayers. We explained the effects of the hydrostatic and biaxial strain on the transition energy when the dots are encapsulated by InGaAs layer.

\section{Experimental Details}

The samples under investigation in this study were grown on semi-insulating, (113)A-oriented GaAs substrates using a Riber $32 \mathrm{MBE}$ system equipped with conventional solidsource diffusion cells and a $12 \mathrm{keV}$ RHEED gun. The substrate native oxides were removed from the surface at grown temperature $580^{\circ} \mathrm{C}$ under an arsenic flux. The GaAs growth rate was set to 0.71 monolayer $(\mathrm{ML}) / \mathrm{s}$. The growth rates were calibrated with Stranski-Krastanov (SK) transition and RHEED oscillations during epitaxial growth of InAs and GaAs layers on GaAs (113)A, respectively. The growth temperature of InAs QDs is fixed at $500^{\circ} \mathrm{C}$. The substrate temperature was measured by an infrared pyrometer. The RHEED pattern was recorded using our newly developed digital image analysis system, which combines a very sensitive CCD camera and a frame grabber as well as dedicated software. The 3 MLs InAs QDs samples were capped by a thin GaAs and $\operatorname{In}_{x} \mathrm{Ga}_{1-x} \mathrm{As}(x=0.3)$ layers, respectively.

An in situ reflection high-energy electron diffraction (RHEED) pattern was used to investigate the formation process of the InAs quantum dots. Photoluminescence measurements were carried out between 10 and $300 \mathrm{~K}$ while keeping the samples in a closed-cycle helium circulation cryostat. The excitation wavelength used is the $514,5 \mathrm{~nm}$ line of the $\mathrm{cw} \mathrm{Ar}{ }^{+}$laser. The emission was dispersed by a highresolution spectrometer and detected by a thermoelectrically cooled InGaAs photodetector with a built-in amplifier. The polarization measurements were performed on the PL emission normal to the surface via a Glan-Thompson near infrared polarizer at the entrance slit of the monochromator followed by a quarter wave plate in order to get the PL signal independent of any light polarization effect of the optical system response.

\section{Results and Discussions}

In order to properly confine the carriers in quantum dots, it is essential that these dots are encapsulated. However, the problem that arises is to choose the material suitable for encapsulation. When encapsulating quantum dots with a few monolayer of GaAs, for example, Ga atoms, diffuse into the dot which reduces their sizes. So, the heart of the dots remains in InAs while the edges are composed of ternary InGaAs. It can modify the structural and optical properties of QDs. However, it is known that the growth of InGaAs layer on InAs quantum dots, developed on (001) GaAs substrates, reduces the stress in quantum dots due to the decrease of hydrostatic strain. This phenomena shifts the emission energy of QDs to the red [8]. But the question that arises here is the following: what have we observed if the InAs QDs are developed on high-index GaAs substrates? To answer this question, we studied two samples, one is encapsulated by GaAs, the other by InGaAs. The photoluminescence spectra of these two structures made at low temperature are shown in Figure 1. For sample covered by GaAs, the PL emission has a inhomogeneous broadening at the low-energy side. The spectrum can be decomposed into two Gaussians: the first is centered on $1.26 \mathrm{eV}$, and the second is located at $1.3 \mathrm{eV}$. Both peaks have the same FWHM $(60 \mathrm{meV})$. To explain the asymmetry of the spectrum, a study of PL as a function of excitation density is required.

Figure 2 shows the variation of PL spectra as a function of excitation density. We note first that the PL intensity increases with excitation density. In addition, we found that the energy position and width at half maximum does not exhibit significant variations. Secondly, we observe that the PL spectra retain their asymmetry of low-energy side, indicating an effect of simultaneous filling of states corresponding to two peaks. We can therefore attribute the two Gaussian peaks (Figure 1) to the two ground states of different quantum dot sizes: small dots with energy emission at $1.3 \mathrm{eV}$ and large dots emitting at $1.26 \mathrm{eV}$. The shape of the spectrum is a direct result of dispersion in quantum dot sizes. PL and AFM have been carried out for InGaAs QDs prepared on high-index GaAs substrates that confirm this size dispersion [8-15]. This is explained by the presence of surface steps in GaAs (113) substrates, which could influence that the diffusion length of adatoms is a key factor in determining the shape, size, and density of QDs. For the sample covered by InGaAs epilayer, we note first an abnormal blue shift with an energy of $35 \mathrm{meV}$ compared to the emission of the sample encapsulated by GaAs. Second, we observe the appearance of another PL band at the low-energy side located at $1.17 \mathrm{eV}$. We have an asymmetry of the two PL bands with an FWHM of about $30 \mathrm{meV}$. This result may be due to the changes of the In compositions in the quantum dot.

These results were predicted theoretically by Mlinar et al. $[8,9]$ which shows that the emission of InAs quantum dot covered by $\operatorname{In}_{x} \mathrm{Ga}_{1-x}$ As and $\mathrm{GaAs}$ substrates prepared on (111), (112), and (113) would be shifted to high energies when certain conditions (size, composition) are to be realized. Mlinar et al. showed that, for small size dots, the fundamental transition decreases with the indium content of whatever substrates orientation, while, for large QD, the transition energy increases to a threshold value, then it decreases for high-index orientations. However, it decreases continuously for the orientation (001). This shift to high energies is in terms of competition between the hydrostatic and biaxial strain [5-11]. Specifically, the hydrostatic strain decreases with the indium concentration for the small size dots, whatever the orientation of the substrate. This results in the monotonic decrease in the energy transition as a function of the indium fraction in $\operatorname{In}_{x} \mathrm{Ga}_{1-x}$ As. However, the biaxial strain is responsible for the decrease of the discontinuity of the valance band (VB), Which increases with the indium composition. In the case of high-index substrates the biaxial strain is greater than the hydrostatic strain, so the transition energy increases when the dots are covered with InGaAs 


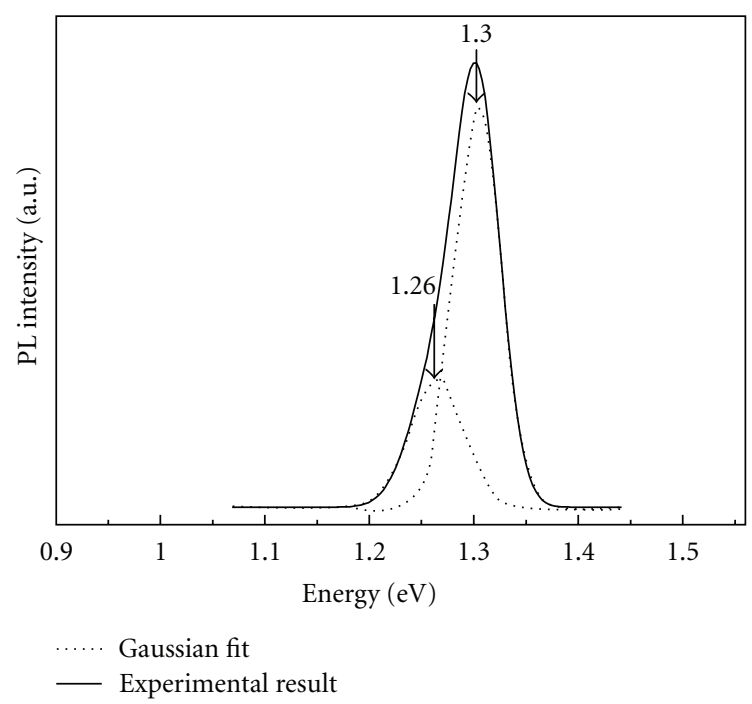

(a)

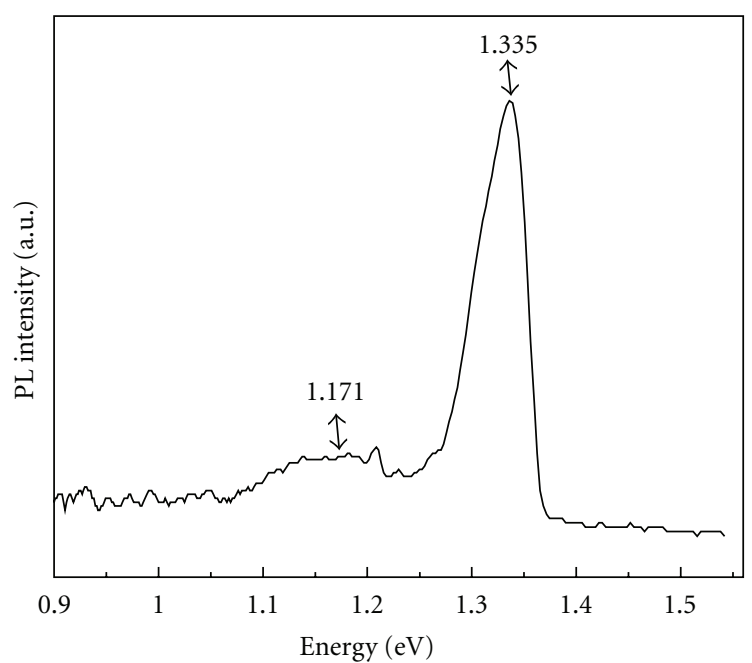

(b)

Figure 1: $10 \mathrm{~K}$ PL spectra of InAs/GaAs (113) quantum dot capped by GaAs epilayer (a) and InGaAs epilayers (b).

which is not the case for the orientation (001) where the hydrostatic strain is the most important $[8,9]$. About width at half maximum of PL spectra, Figure 1 shows that this parameter decreases from 60 to $30 \mathrm{meV}$ when the dots are capped by the InGaAs. This decrease reflects an improvement in uniformity of quantum dots.

These results are explained by the fact that, for small quantum dots L1, when they are encapsulated by InGaAs, their biaxial strain increases which enhances their emission energy. However, large quantum dots, when they are encapsulated by InGaAs, their hydrostatic strain decreases, which decreases their emission energy.

Actually, the competition between the increased biaxial component of the strain tensor, responsible for the decrease of the valence band mixing and the decrease of the hydrostatic strain with increase of the In concentration, determines

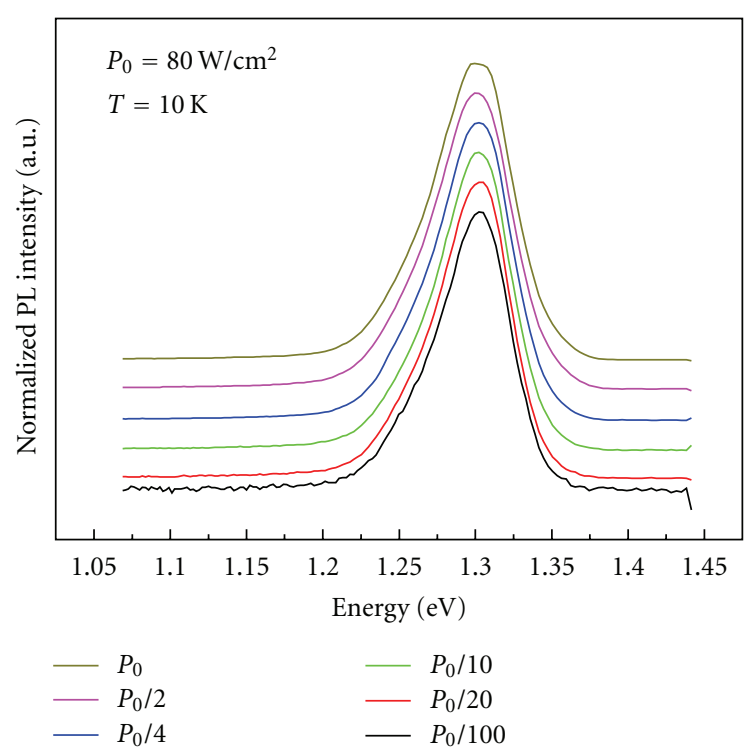

FIGURE 2: Evolution of PL spectra as a function of excitation density of InAs QDS capped by GAAs epilayers.

the transition energy. Note also that even for [001] grown small QD size, biaxial strain is increased, but the hydrostatic strain has a dominant influence on the transition energy $[8,9]$.

In fact, a broadening of the PL emission due to size distribution of the dots, when InAs dots are capped by GaAs, is observed. A separation between large and small quantum dots, when they are encapsulated by InGaAs, is due to hydrostatic and biaxial strain action on large and small dots grown under specifically growth conditions.

PL polarization measurements were performed in the linear response regime. The degree of linear polarization $P$ of the emitted light is classically defined by the following equation:

$$
P=\frac{I_{[33-2]}-I_{[-110]}}{I_{[33-2]}+I_{[-110]}}
$$

where $I_{[33-2]}$ and $I_{[-110]}$ are the PL intensities along the two orthogonal directions [33-2] and [-110].

No PL polarization anisotropy is observed $(P \sim 0 \%)$ for InAs quantum dots capped with GaAs layer (Figure 3(a)) which is due to the dispersion in size and shape of the quantum dots. Indeed, the luminescence is identical in all directions because of the dispersion in size and shape of the dots. On the InAs QDs capped by InGaAs sample, a small anisotropy is observed $(P \sim 8 \%)$ for the high-energy side since the PL peak intensities observed in the $[-110]$ direction of the polarizer are smaller than those observed in the [33-2] direction. The degree of linear polarization is found to be around $10 \%$ (Figure $3(\mathrm{~b})$ ). This result is the clear signature of elongated quantum dots [16-18]. Although the small dots require an elongated form, the large dots present a quasiisotropic behavior. 


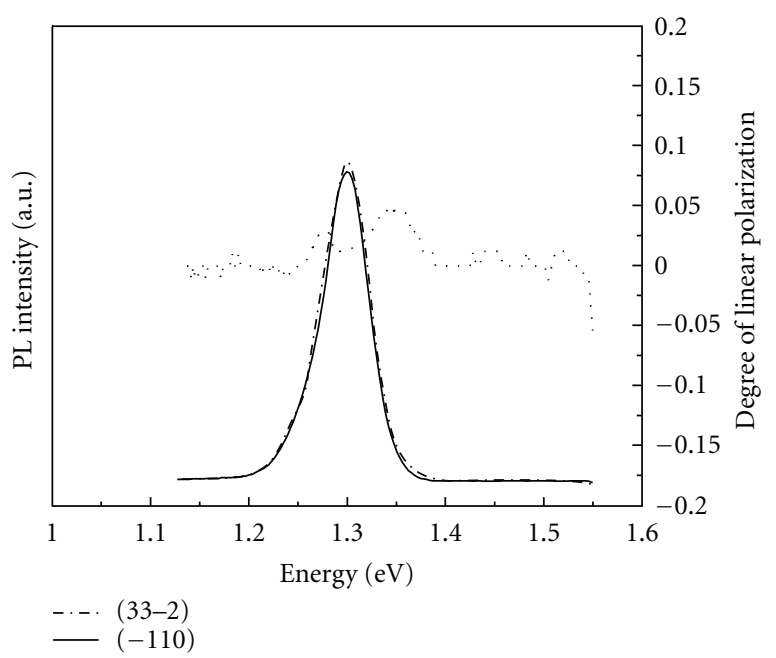

(a)

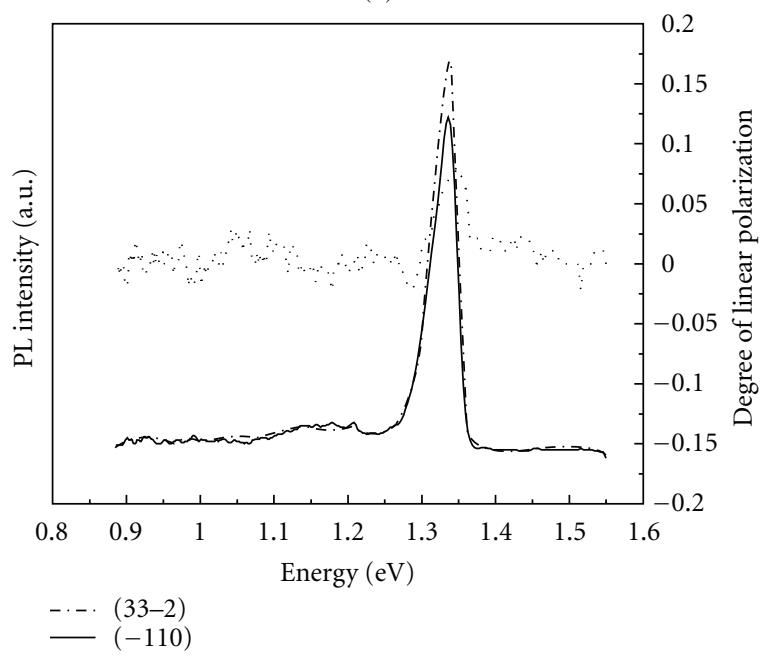

(b)

Figure 3: Polarization PL spectra at $10 \mathrm{~K}$ and degree of linear polarization for the InAs capped by GaAs sample (a) and InGaAs sample (b).

\section{Conclusion}

We have studied optical properties of InAs/GaAs (113)A quantum dots grown by molecular beam epitaxy (MBE) capped by $(\mathrm{In}, \mathrm{Ga})$ As. A broadening of the PL emission due to size distribution of the dots, when InAs dots are capped by GaAs, was observed. A separation between large and small quantum dots, when they are encapsulated by InGaAs, has been shown due to hydrostatic and biaxial strain action on large and small dots grown under specific growth conditions. The PL polarization measurements have been investigated. We have shown that the small dots require an elongated form but the large dots present a quasi-isotropic behavior.

\section{References}

[1] M. Schmidbauer, Sh. Seydmohamadi, D. Grigoriev et al., "Controlling planar and vertical ordering in three-dimensional
(In,Ga)As quantum dot lattices by GaAs surface orientation," Physical Review Letters, vol. 96, no. 6, Article ID 66108, 4 pages, 2006.

[2] P. Caro, C. Paranhoen, C. Platz et al., "High-gain and lowthreshold InAs quantum-dot lasers on InP," Applied Physics Letters, vol. 87, no. 24, Article ID 243107, 3 pages, 2005.

[3] C. Çelebi, J. M. Ulloa, P. M. Koenraad, A. Simon, A. Letoublon, and N. Bertru, "Capping of InAs quantum dots grown on (311)B InP studied by cross-sectional scanning tunneling microscopy," Applied Physics Letters, vol. 89, no. 2, Article ID 023119, 2006.

[4] M. C. Xu, Y. Temko, T. Suzuki, and K. Jacobi, "Shape transition of self-assembled InAs quantum dots on GaAs(114)A," Physical Review B, vol. 71, no. 7, Article ID 075314, 8 pages, 2005.

[5] C. H. Lin, W.-W. Pai, F. Y. Chang, and H. H. Lin, "Comparative study of InAs quantum dots with different InGaAs capping methods," Applied Physics Letters, vol. 90, no. 6, Article ID 063102, 3 pages, 2007.

[6] Q. Gong, P. Offermans, R. Nötzel, P. M. Koenraad, and J. H. Wolter, "Capping process of InAs/GaAs quantum dots studied by cross-sectional scanning tunneling microscopy," Applied Physics Letters, vol. 85, no. 23, pp. 5697-5699, 2004.

[7] R. H. Henderson and E. Towe, "Strain and crystallographic orientation effects on interband optical matrix elements and band gaps of [111 ]-oriented III-V epilayers," Journal of Applied Physics, vol. 78, no. 4, pp. 2447-2455, 1995.

[8] V. Mlinar and F. M. Peeters, "Influence of the substrate orientation on the electronic and optical properties of InAs/GaAs quantum dots," Applied Physics Letters, vol. 89, no. 26, Article ID 261910, 3 pages, 2006.

[9] V. Mlinar, M. Tadić, B. Partoens, and F. M. Peeters, "Nonsymmetrized Hamiltonian for semiconducting nanostructures in a magnetic field," Physical Review B, vol. 71, no. 20, Article ID 205305, pp. 1-12, 2005.

[10] J. H. Lee, Z. M. Wang, and G. J. Salamo, "The control on size and density of InAs QDs by droplet epitaxy (april 2009)," IEEE Transactions on Nanotechnology, vol. 8, no. 4, Article ID 4907084, pp. 431-436, 2009.

[11] T. Mano, T. Kuroda, K. Mitsuishi, T. Noda, and K. Sakoda, "High-density GaAs/AlGaAs quantum dots formed on GaAs (3 1 1)A substrates by droplet epitaxy," Journal of Crystal Growth, vol. 311, no. 7, pp. 1828-1831, 2009.

[12] E. Stock, T. Warming, I. Ostapenko et al., "Single-photon emission from InGaAs quantum dots grown on (111) GaAs," Applied Physics Letters, vol. 96, no. 9, Article ID 093112, 2010.

[13] L. Bouzaïene, F. Saidi, L. Sfaxi, and H. Maaref, “Temperature dependence of the optical properties of InAs quantum dots with bimodal size evolution grown on GaAs ( $\left.\begin{array}{lll}1 & 1 & 5\end{array}\right) \mathrm{A}$ substrate," Physica B, vol. 405, no. 2, pp. 744-747, 2010.

[14] L. Sfaxi, L. Bouzaiene, H. Sghaier, and H. Maaref, "Effect of growth temperature on InAs wetting layer grown on (llll 113$) A$ GaAs by molecular beam epitaxy," Journal of Crystal Growth, vol. 293, no. 2, pp. 330-334, 2006.

[15] J. H. Lee, Z. M. Wang, E. S. Kim, N. Y. Kim, S. H. Park, and G. J. Salamo, "Self-assembled InGaAs tandem nanostructures consisting of a hole and pyramid on GaAs (311) A by droplet epitaxy," Physica Status Solidi A, vol. 207, no. 2, pp. 348-353, 2010.

[16] L. M. Kong, J. F. Cai, Z. Y. Wu, Z. Gong, Z. C. Niu, and Z. C. Feng, "Time-resolved photoluminescence spectra of selfassembled InAs/GaAs quantum dots," Thin Solid Films, vol. 498, no. 1-2, pp. 188-192, 2006. 
[17] Y. Furukawa and S. Noda, "Difference of anisotropic structures of InAs/GaAs quantum dots between organometallic vaporphase epitaxy and molecular beam epitaxy," Journal of Crystal Growth, vol. 220, no. 4, pp. 425-431, 2000.

[18] Y. Chen, Bo. Xu, F. Liu, L. Shi, C. Tang, and Z. Wang, "Polarization dependence of absorption in strongly vertically coupled InAs/GaAs quantum dots for two-color far-infrared photodetector," Physica E, vol. 40, no. 3, pp. 633-636, 2008. 


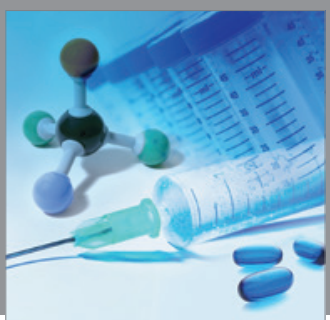

International Journal of

Medicinal Chemistry

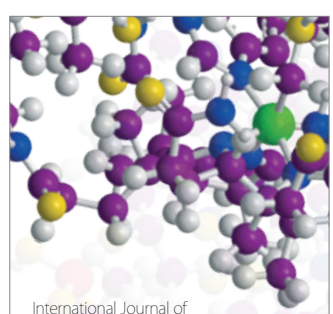

Carbohydrate Chemistry

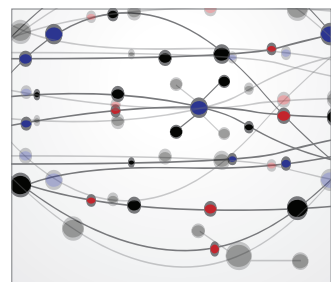

The Scientific World Journal
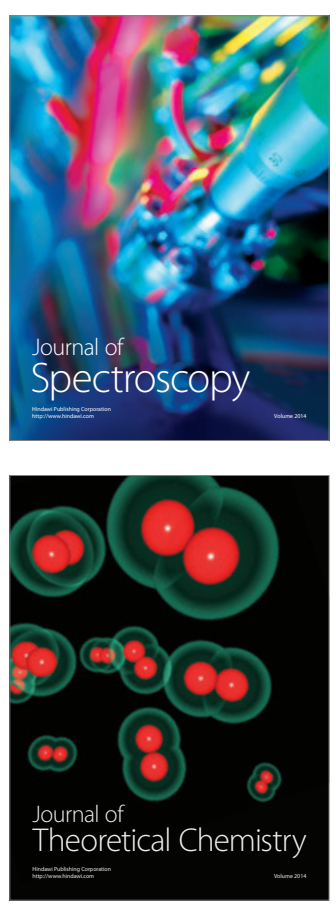
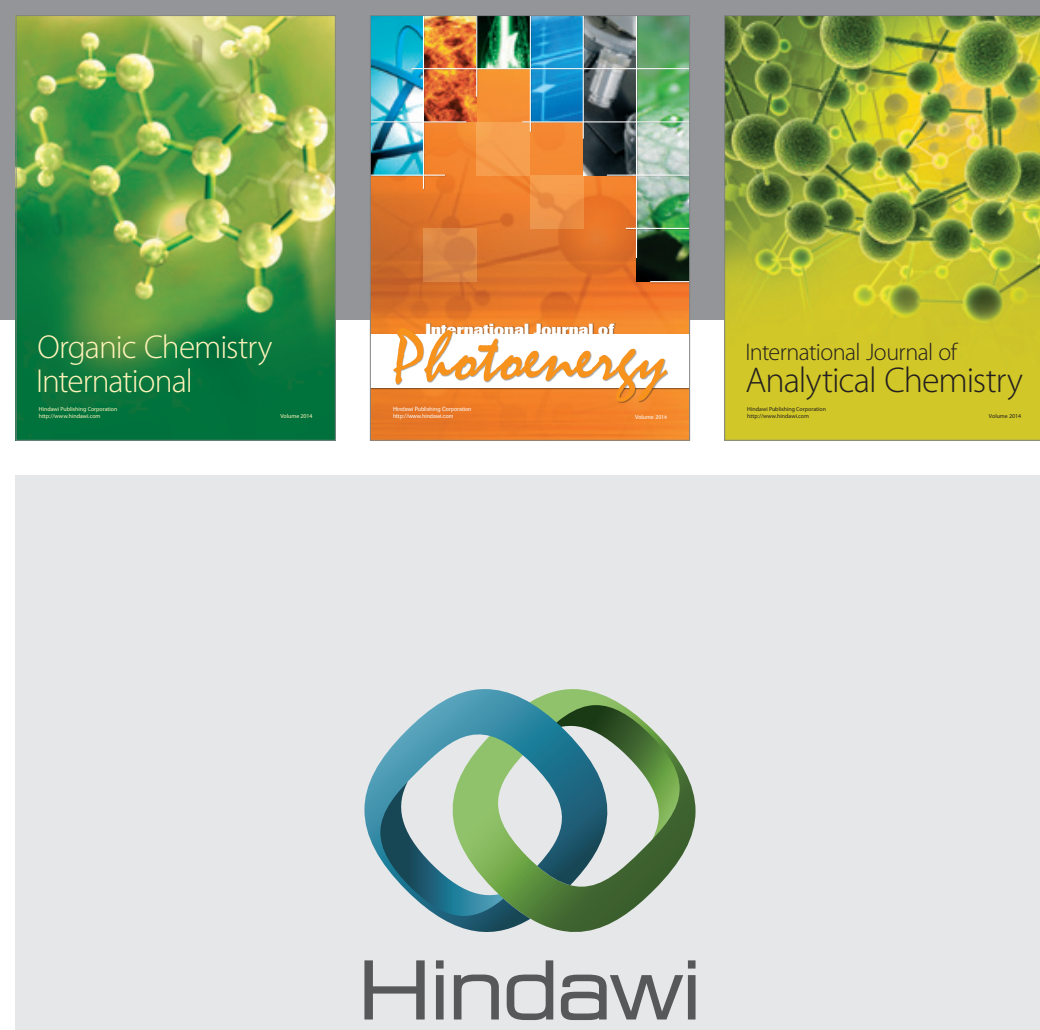

Submit your manuscripts at

http://www.hindawi.com
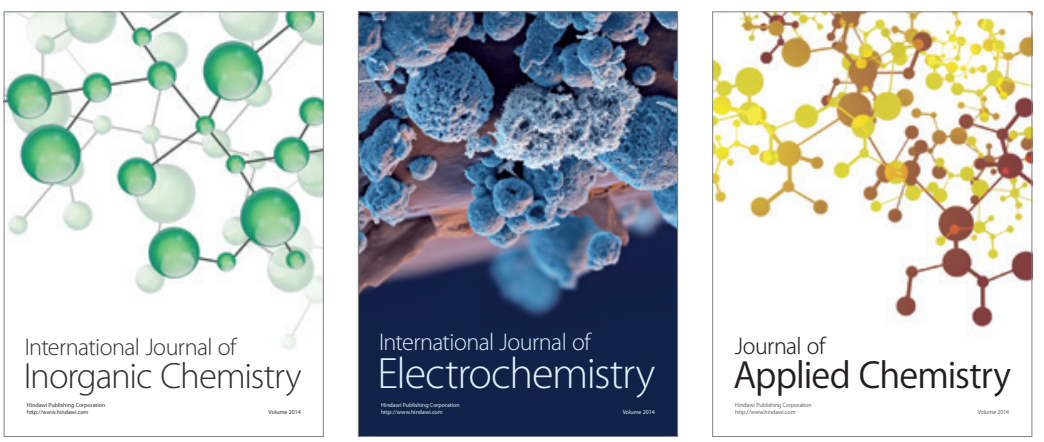

Journal of

Applied Chemistry
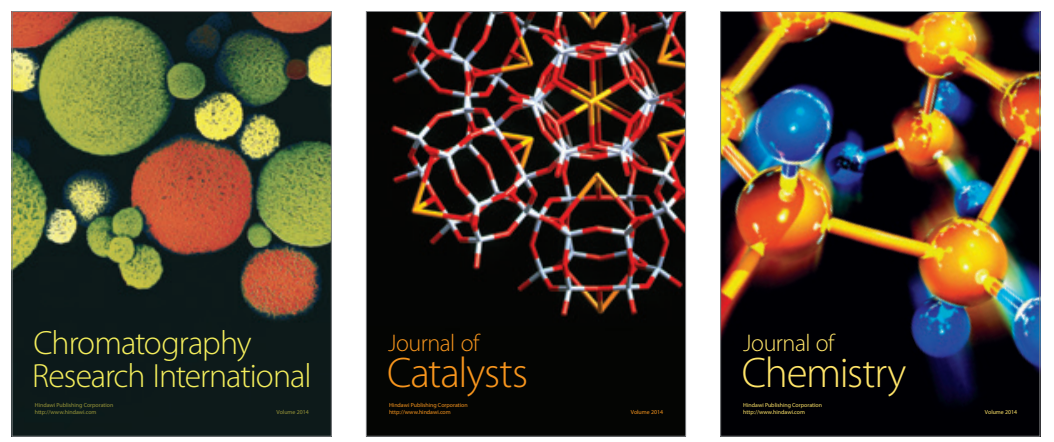
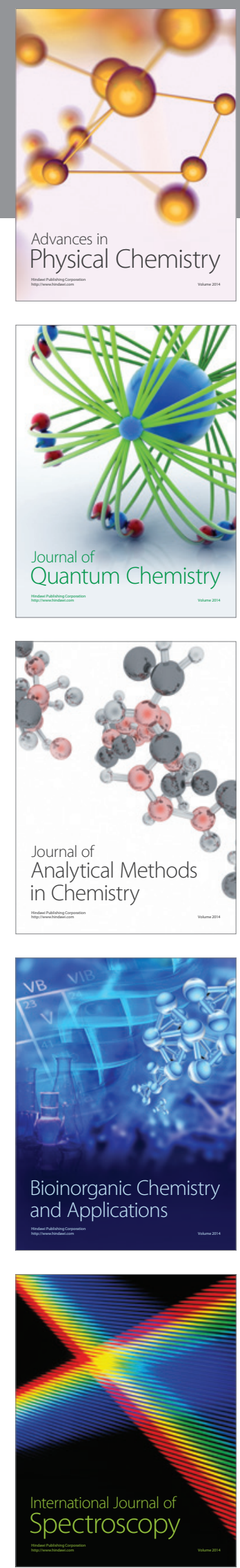